\title{
Topic modeling reveals distinct posting patterns within an online conspiracy forum.
}

\author{
Colin Klein ${ }^{1,2^{*} \ddagger}$, Peter Clutton ${ }^{1,2 \ddagger}$, Vince Polito ${ }^{2,3 \ddagger}$,
}

1 Department of Philosophy, Macquarie University, Sydney, NSW 2109,Australia 2 ARC Centre of Excellence in Cognition and its Disorders, Macquarie University, Sydney, NSW 2019,Australia 3 Department of Cognitive Science, Macquarie University, Sydney, NSW 2109,Australia

$\ddagger$ These authors contributed equally to this work.

* colin.klein@mq.edu.au 


\section{Abstract}

Conspiracy theories play a troubling role in political discourse, yet the motivations of conspiracy endorsers can be obscure to outsiders. Online forums provide a valuable window into the day-to-day discourse of conspiracy theorizing, but one that is difficult to quantify. We used non-negative matrix factorization to create a topic model of the $r$ /conspiracy forum on Reddit.com. This subreddit provides a large and unique corpus which spans many years and numerous authors. We show that within the forum, there are multiple sub-populations distinguishable by their topic loading. Further, these differences are readily interpretable as differences in background beliefs and motivations. These groups regularly interact with one another by commenting; hence topic modeling reveals subgroups that would not be revealed by simple network analyses. The diversity of the distinct subgroups places constraints on theories of what generates conspiracy theorizing: neither simple irrationality nor common preoccupations of topics seem to fit the bill. Instead, we suggest, conspiracy theorizing seems to be primarily driven by higher-order preoccupations with evidence and argument, along with rhetorical strategies which allow diverse groups to each address any particular theme.

\section{Introduction}

Alexis de Tocqueville wrote that the American commitment to freedom of association prevented the formation of conspiracies [1]. Considering recent political discourse, this is perhaps optimistic; indeed, conspiracy theorizing has long been a part of American politics [2]. The free association promoted by online forums forms a key mechanism for the spread of misinformation, including conspiracy theories $[3 \sqrt{5}$. Conspiracy theories are not socially innocuous: aside from obvious political effects, endorsement of conspiracy theories is associated with rejection of science and unwillingness to donate to prosocial causes [6].

Numerous psychological accounts purport to explain the attraction of conspiracy theories. Conspiracy endorsers are said to favor a 'monological' belief system, in which every event is connected to every other event [6, 7]; to be driven by feelings of powerlessness and lack of control [8]; to crave explanation in a fundamentally confusing world [9]; to be motivated by background political beliefs [10]; to seek social individuation [11]; or to be misled by the 'echo chambers' of online opinion [5, 12].

Experimental studies [13] give some insight into the personality structure of conspiracy endorsers. Yet most such studies use a convenience population of students and artificial tasks with questionable ecological validity. Examination of online comments to news stories [14 gives more insight into 
the public arguments of committed conspiracy theorists, but it is unclear ${ }_{22}$ how widely this generalizes. Only the most vocal and colorful conspiracy ${ }_{23}$ theorists are likely to come to public attention, obscuring the larger but ${ }_{24}$ more circumspect population who merely endorse conspiracy theories. 25

Finally, many studies assume that 'conspiracy theorist' picks out some $\quad 26$ kind of relatively homogeneous group whose behavior is driven by the ${ }_{27}$ same principles. So for example researchers ask 'who tends to believe 28 [conspiracy theories], and why' [15] or inquire into the 'psychological origin' 29 of conspiracy theories [16], or what ask about the 'similar underlying 30 psychological processes' that are common to conspiracy believers [17]. In 31 each case the implication is that there is a common psychological factor 32 which characterizes conspiracy endorsers and distinguishes them from others. 33

Yet as Wood et al [14] have noted, there are serious disagreements and 34 arguments even within conspiracy communities. Conspiracy theorists debate 35 every aspect of (e.g.) the 9/11 attacks, with the only point of agreement 36 being that the official story is (somehow) false. This heterogeneity can be ${ }_{37}$ difficult to quantify. Yet accounting for diversity as well as similarity is 38 vital for any account that wants to show the underpinnings of conspiracy 39 endorsement.

This study presents a novel way to investigate and quantify heterogeneity within a large group of conspiracy endorsers. We used a large publicly available collection of online comments from the r/conspiracy forum on the website reddit.com. Reddit is a collection of online forums ('subreddits'), most of which are user-run and moderated. Reddit is a popular site, with web traffic consistently ranking in the top 10 US sites. While most forums are comparatively anodyne, Reddit's historical commitment to freedom of speech has allowed fringe communities to flourish. Reddit makes its comments available to researchers through a public API, and a comprehensive dataset covering nearly 8 years of comments is available.

Topic modeling is a method for representing each document in a corpus as being generated by a variety of distinct topics, each of which consists of a weighted set of words. Topic modeling techniques have become popular because they allow unsupervised dimensionality reduction: much of the variability in a large corpus like the $r$ /conspiracy comments can be captured by a relatively small number of topics. The topics generated tend to be easily interpretable by experimenters. Documents (in our case, collections of posters' comments) are represented as a mix of different topics, allowing for fine-grained categorization and analysis.

As topic modeling has its roots in automated information retrieval, most $\quad 60$ uses focus on the categorization of individual documents by sorting them ${ }_{61}$ into semantically similar clusters for later retrieval and analysis. However, ${ }_{62}$ one can equally well use topic modeling to explore the psychological moti- ${ }_{63}$ vations of the authors who make the comments [18]. That is our approach: ${ }_{64}$ 
having fitted a topic model to $r$ /conspiracy, we propose to interpret it as ${ }_{65}$ an (abstract and idealized) model of the underlying psychological processes $\quad 66$ of the authors involved. Topic modeling, combined with detailed descrip- 67 tive statistics about posting patterns, can thus reveal something about ${ }_{68}$ the underlying motivations and belief formation processes of conspiracy 69 endorsers. We thus predicted that $\mathrm{r}$ /conspiracy would contain subgroups 70 that differed in meaningful ways on their topic loadings and other measures $\quad 71$ of interest.

\section{Materials and methods}

\section{Dataset and preprocessing}

We used a publicly available dataset containing 1.7 billion comments and associated metadata spanning from October 2007 to May 2015. This includes nearly 2.25 million comments to $r$ /conspiracy, made by nearly 130,000 distinct authors. Reddit posts consist of an initial 'link' posttypically just a link to another website - followed by nested comments underneath. Only comments are included in the dataset and the analysis. Comments outnumber link posts by roughly 12:1, and link posts rarely contain more than a URL; of the top 10 most commented-upon links in the dataset, only one contained substantial original text.

We removed high-posting bots, pre-processed the text to remove common words and non-linguistic data, and combined each author's comments into a single document [19 21]; S1 Appendix contains full details. The result was a term frequency-inverse document frequency (tf-idf) representation of each author's combined contributions to the $r /$ conspiracy forum.

\section{Topic modeling}

To analyze the linguistic structure of the data, we constructed a topic 90 model, using the combined author comments as the documents of the target $9_{91}$ corpus. We used non-negative matrix factorization (NMF), an algorithm 92 developed to find common parts of pictures and which is well-suited to ${ }_{93}$ corpus analysis $[22[24]$.

The overall choice of topic number depends on one's theoretical concerns 25. Our goal was to find common themes hidden among a group of superficially similar commenters. This argued for a comparatively small number of topics compared to uses which seek to make fine-grained classifications of documents. Choosing too small a topic number tends to 99 result in topics that overlap and run together different themes. To optimise ${ }_{100}$ between these two demands, we examined a quantifiable measure of the ${ }_{101}$ similarity of the derived topics to one another for a range of topic models. ${ }_{102}$ 
We constructed an NMF model for $t=12,15, \ldots 90$ and then calculated the ${ }_{103}$ mean cosine similarity between each pair of topics in the generated model. ${ }^{104}$ We chose $t=48$ as the point where the mean cosine similarity began to ${ }_{105}$ flatten out (see S1 Fig). The full 48-topic NMF model used for subsequent ${ }_{106}$ analyses is shown in S1 Table.

\section{Subgroup clustering and statistics}

We predicted that $r$ /conspiracy would contain subgroups of authors whose 109 posts differed in meaningful ways in their loadings on the extracted topics. ${ }_{110}$ To test this hypothesis, we categorized the authors in $r$ /conspiracy into ${ }_{111}$ subgroups by using authors' loadings in the document-topic mapping as the ${ }_{112}$ basis for a k-means clustering. Authors who made only one or two posts ${ }_{113}$ were eliminated from the set, as preliminary analysis showed that they ${ }_{114}$ only added to noisy clusterings. To chose a number of clusters, we then ${ }_{115}$ performed kmeans clustering from $n=2 . .30$ and calculated the percentage ${ }_{116}$ of variance explained (PVE) for each. The improvement in PVE begins ${ }_{117}$ to level out around $n=12$ (see S2 Fig. To further ensure that $n=12{ }_{118}$ was a reasonable cluster solution, repeated Linear Discriminant Analysis ${ }_{119}$ (LDA) was used to verify cluster discriminability. Data and labels were ${ }_{120}$ shuffled and an LDA model fitted on half and tested on the other half. ${ }_{121}$ This procedure was repeated 100 times. The resulting mean classification 122 accuracy of $89 \%$ (SD .002) is significantly different from expected chance ${ }_{123}$ performance of $1 / 12 \approx 8 \%(p<.001)$ suggesting that the derived subgroups ${ }_{124}$ do pick out stable clusters in topic space.

For the entirety of $r$ /conspiracy and each of the 12 subgroups, we ${ }_{126}$ calculated number of comments, number of authors, mean number of ${ }_{127}$ comments by author, and the post concentration, which we define as the ${ }_{128}$ percentage of authors who were responsible for the top $50 \%$ of comments. ${ }^{129}$ In addition to basic descriptive statistics, we examined four other measures $\quad{ }_{130}$ to characterize the posting patterns of the subgroup relative to the whole. ${ }^{131}$

First, we compared the posts that each author made in other subreddits ${ }_{132}$ versus the number of posts they made in $r$ /conspiracy (the external to ${ }_{133}$ target (E:T) ratio). The lower the E:T ratio, the more authors tend to ${ }_{134}$ concentrate on the target forum alone. Second, to examine the degree to 135 which authors tend to post with others in their group, we calculated the ${ }_{136}$ mean link diversity (MLD) for each subgroup. MLD measures how many ${ }_{137}$ authors from other subgroups, on average, post to threads in r/conspiracy 138 that authors in a subgroup post to. MLD can range from 1 (authors in ${ }_{139}$ the subgroup post only with authors in their own subgroup) to 12 (authors 140 post in threads with representatives from every other subgroup.) A low ${ }_{141}$ MLD would suggest that subgroups with different language use patterns ${ }_{142}$ self-segregate within the forum, while a high MLD suggests the opposite. ${ }^{143}$ 
Third, to characterize the posting patterns of the subgroups relative ${ }_{144}$ to the whole, we examined loadings by topic for each of the subgroups. ${ }_{145}$ In test for variation between subgroups, we selected by determining the ${ }_{146}$ standard deviation of the loadings by subgroup and then choosing the $15{ }_{147}$ with the highest standard deviation. For topic loadings, significance level ${ }_{148}$ of $p<0.01$ (uncorrected) was determined by a Monte Carlo method. The ${ }_{149}$ full document-topic mapping was shuffled and subgroups the size of the ${ }_{150}$ smallest subgroup were randomly selected. This was repeated 1000 times 151 for each topic, and used to create upper and lower bounds for significance ${ }_{152}$ per topic.

Fourth and finally, we examined the correlation between a users' loading on topics and the logarithm of each users' combined overall comment length (by character count, which correlates highly with both word count $(\mathrm{r}=0.99)$ and number of comments made by the author $(\mathrm{r}=0.91)$ ). The logarithm was ${ }_{157}$ used as comment length varies over several orders of magnitude. Because ${ }_{158}$ NMF fits the tf-idf representation rather than absolute word counts, a 159 positive correlation between a topic and length should indicate that an ${ }_{160}$ author uses the words in the topic more frequently and distinctively the ${ }_{161}$ more they write. However, there is also a general correlation between topic ${ }_{162}$ loading and length across all topics ( due in part to general properties of ${ }_{163}$ the tf-idf matrix, and in part to the fact that NMF picks words that tend ${ }_{164}$ to be more frequently represented in larger topics). To account for this ${ }_{165}$ general trend, we generated 10,000 pseudo-topics by randomly selecting one ${ }_{166}$ of the 48 loadings for each author, and used this to calculate the mean and ${ }_{167}$ 95\% uncorrected confidence interval for the correlation between loading ${ }_{168}$ and $\log$ length $(r=0.21 \pm 0.005)$. Observed correlations above or below ${ }_{169}$ this were considered significantly different.

\section{Aggregate comments and tags ${ }_{171}$}

To facilitate discussion, we assigned each subgroup a short name based 172 on their distinctive posting patterns. In order to create a representative ${ }_{173}$ but anonymous sample comment, we chose comments from the top 2-4 174 posters in each subgroup, aggregating representative comments. We altered 175 the grammar and word choice enough that a search would not reveal the ${ }_{176}$ original posters. Each poster has considerable variability in their own posts, 177 $\begin{array}{ll}\text { but the quotes are representative of the whole. } & 178\end{array}$

Subgroup 11, the 'uncategorized' group, contains a mix of low-posting 179 contributors, missed bots, and other difficult-to-categorize posts. There ${ }_{180}$ is undoubtedly further structure in this group which might be pulled out ${ }_{181}$ with further analysis, but much of it is clearly a noise cluster. 182 


\section{Results}

\section{Topic loadings}

The top of Fig 1 shows the mean loadings by subgroup on the 15 most vari- 185 able topics. Mean topic loadings reveal substantial between-subgroup differ- ${ }_{186}$ ences, as well as differences between individual subgroups and $r$ /conspiracy $\quad{ }_{187}$ as a whole. The bottom of Fig 1 shows a descriptive tag and aggregate ${ }_{188}$ comment for each group. The descriptive tags will be used in what follows, 189 placed in small caps to emphasize that they are convenience labels. $\quad 190$

In general, three patterns are seen. First, there are the two sets of 191 True Believers, which load highly on most topics. Members of these ${ }_{192}$ subgroups also tend to be frequent and enthusiastic posters, as shown by ${ }_{193}$ the posting statistics. Comment inspection suggests differences between 194 these subgroups - very roughly, group 3 cares more about historical events $\quad 195$ while group 7 is willing to entertain more speculative conspiracies involving 196 things like UFOs - but we will consider them together.

Second, there are groups who are distinguished by very high loadings 198 on a single topic and average/low loadings on the others. Some of these 199 are what we will call traditional 'thematic' concerns: AnTI-SEMITES are 200 concerned with Jews and Israel; PATRIOTs by constitutional issues, and 201 especially gun control; TRUTHERS by conspiracy theories around the 9/11 202 attacks; ANTI-Authoritarians by police use of force against civilians, 203 and Anti-Imperalists by the consequences of American power more 204 generally.

Also of note in this pattern are the Pseudo-SCIEnTISTs, who are con- 206 cerned with quasi-scientific topics such as anti-vaccination advocacy or ${ }_{207}$ chemtrails. This subgroups brings up an important point: topic modeling 208 does not distinguish between those who endorse a conspiracy and those ${ }_{209}$ who argue with conspiracy theorists. Further, there is considerable debate ${ }_{210}$ even among TRUE BELIEvers about which conspiracy theories are worth ${ }_{211}$ endorsing. While this is an important caveat, it is a minor one. For one, 212 comment inspection reveals that most subgroups are primarily composed ${ }_{213}$ of endorsers: those who are generally unimpressed by conspiracy theories 214 are lumped by the algorithm into the SkePtics. For another, the fact that ${ }_{215}$ there are linguistically separable patterns of argument is itself interesting. ${ }^{216}$

The third and final pattern was less expected. There are groups who are ${ }_{217}$ distinguished by more 'rhetorical' topics. The most notable among these ${ }_{218}$ are the INDIGNANT, which are distinguished by high loading on a topic ${ }_{219}$ consisting of angry invective. What distinguishes the INDIGNANT in other 220 words, is not what they talk about but how they talk about it. Similarly, 221 REDDITORS are marked by discussion of Reddit itself and associated social 222 drama, rather than particular themes.

Turning to the topics themselves, topics like 3, 5, 14, and 17 also 224 
appear to be primarily rhetorical in nature. Loadings on these topics vary 225 across groups, albeit less dramatically. These topics seem to contain words 226 that play an important role in mediating ingroup identification along with ${ }_{227}$ agreement and disagreement, suggesting that such mediation is differentially ${ }_{228}$ important for different groups.

\section{Topic correlations}

Fig 2 shows the correlation between topic loadings and log comment length. 231 (Raw numbers can be found in S2 Table). This shows which topics become ${ }_{232}$ more important the more people post. These results largely dissociate from 233 the picture provided by the pure topic loadings. Consider, for example, 234 the Anti-SEMites, who load heavily on the two topics having to do with 235 Judaism. The correlation with log length and these two topics is only ${ }_{236}$ slightly above average, as you might expect: group membership is defined ${ }_{237}$ in part by placing a high importance on these topics no matter how often 238 one posts. On the other hand, ANTI-SEMITES show a high loading with ${ }_{239}$ (e.g.) topic 13, suggesting that 9/11 conspiracy theories become more 240 important for this group the more they write. Conversely, the opposite 241 pattern is also shown-most subgroups are slightly less likely than average 242 to talk about gun control the more they write. 243

Along the rows, two striking patterns emerge. Topic 0, which concerns 244 evidence, belief, and argument, has the strongest correlations of the group, 245 ranging as high as 0.75 in the subgroups and 0.73 overall (numeric scores 246 are depicted in S2 Table). Topic 34, concerning American military power ${ }_{247}$ and foreign policy, has a similarly high pattern of correlation (as does topic 248 35 , not pictured here).

\section{Posting statistics}

Table 1 shows basic statistics for number of authors and posts by subgroup 251 and for the whole. These vary quite widely: the TRUE BELIEVERS are only ${ }_{252}$ $5 \%$ of the authors in $\mathrm{r}$ /conspiracy but responsible for $64 \%$ of the total ${ }^{253}$ comments.

Table 2 shows data about the External/Target statistics and the Mean 255 Neighborhood Diversity for both the group as a whole and each subgroup. 256 Two important trends are worth noting. First, there is wide variability ${ }_{257}$ in E:T ratios, with posters in the most enthusiastic subgroups showing a 258 relatively low E:T. That said, even the group with the lowest average E:T ${ }_{259}$ still makes on average 9 comments in other forums for every one they make ${ }_{260}$ in $r$ /conspiracy. 261

Second, table 1 shows that the MLD for each group and as a whole is 262 relatively high. On average, people post in threads with representatives ${ }_{263}$ from at least 6 other subgroups. MLD ranges only slightly higher or lower. ${ }^{264}$ 
Table 1. Basic Descriptive statistics.

\begin{tabular}{|l|r|r|r|r|r|}
\hline Group & Posts & P/T & Authors & A/T & P/A \\
\hline r/Conspiracy & 2253494 & 1.00 & 129829 & 1.000 & 17.36 \\
\hline Skeptics & 21131 & 0.01 & 2003 & 0.02 & 10.55 \\
Anti-Imperalists & 111601 & 0.05 & 7625 & 0.06 & 14.64 \\
Anti-Authoritarians & 34700 & 0.02 & 2311 & 0.02 & 15.02 \\
True Believers 1 & 531056 & 0.24 & 3650 & 0.03 & 145.49 \\
Patriots & 13986 & 0.01 & 880 & 0.01 & 15.89 \\
Truthers & 57907 & 0.03 & 1268 & 0.01 & 45.67 \\
Psuedoscientists & 163217 & 0.07 & 6523 & 0.05 & 25.02 \\
True Believers 2 & 910116 & 0.40 & 2324 & 0.02 & 391.62 \\
Anti-Semites & 92532 & 0.04 & 1453 & 0.01 & 63.68 \\
Indignant & 47196 & 0.02 & 3011 & 0.02 & 15.67 \\
Redditors & 45091 & 0.02 & 3108 & 0.02 & 14.51 \\
Uncategorized & 124299 & 0.06 & 19146 & 0.15 & 6.49 \\
\hline
\end{tabular}

Descriptive statistics for $r /$ conspiracy and each subgroup. $\mathrm{P} / \mathrm{T}=$ posts by group as percentage of total posts in $\mathrm{r} /$ conspiracy. $\mathrm{A} / \mathrm{T}=$ authors as percentage of total authors., $\mathrm{P} / \mathrm{A}$ posts per author.

Table 2. Descriptive statistics for External posts versus target posts.

\begin{tabular}{|l|r|r|}
\hline Group & E:T & MLD \\
\hline r/Conspiracy & 85 & 7.2 \\
\hline Skeptics & 195 & 7.3 \\
Anti-imperalists & 129 & 7.4 \\
Anti-authoritarians & 121 & 8.4 \\
True Believers 1 & 21 & 6.4 \\
Patriots & 135 & 8.2 \\
Truthers & 51 & 7.7 \\
Psuedoscientists & 81 & 6.9 \\
True Believers 2 & 9 & 5.9 \\
Anti-Semites & 30 & 7.3 \\
Indignant & 126 & 7.7 \\
Redditors & 140 & 7.2 \\
Uncategorized & 254 & 7.1 \\
\hline
\end{tabular}

$\mathrm{E}: \mathrm{T}=$ Mean external to mean target ratio, $\mathrm{MLD}=$ Mean link diversity by group. r/Conspiracy $=$ overall means, subgroups labels from figure 1 .

This suggests that subgroups identified on the basis of topic modeling are ${ }_{265}$ not formed because a single forum hosts non-interacting sub-communities. ${ }_{26}$ 
Despite diversity of interests and language use, it appears that everyone ${ }_{267}$ talks to most everyone else.

\section{Discussion}

\section{Posting patterns}

We began with the claim that conspiracy forums are characterized by impor- ${ }^{271}$ tant diversity among posters, and that it would be rash to over-generalize 272 from the behavior of the most frequent or enthusiastic posters. Our data ${ }_{273}$ provide support for this hypothesis. First, and most obviously, we have 274 shown that commenters in $\mathrm{r}$ /conspiracy fall into meaningful subgroups 275 with distinctive patterns of language use that appear to correspond to 276 distinct concerns.

One would come away with a very different picture if one focused on 278 comments rather than users. As noted before, True BeliEvers are ${ }_{279}$ responsible for a disproportionate number of comments. The top author of 280 the True Believers wrote a staggering 896,337 words (post-processing) ${ }_{281}$ across over 18,000 posts - roughly twice the combined length of the Lord ${ }_{282}$ of the Rings trilogy. Posters in this group may come closest to the classic 283 picture of a 'monological' belief system. 284

However, True Believers represent only about $5 \%$ of the authors in 285 $r /$ conspiracy; as the descriptive statistics show, they also tend to post ${ }_{286}$ voluminously elsewhere on Reddit, suggesting a commitment to posting ${ }_{287}$ rather than to conspiracy theories as such. 288

Most posters in $r$ /conspiracy do not appear to post obsessively within 289 the forum: most post more elsewhere, including the high-volume posters. 290 The sheer number of authors in the dataset should also give pause to 291 anyone proposing simplistic explanations: the posts and volumes in our ${ }_{292}$ dataset are roughly comparable to those in other subreddits such as ${ }_{293}$ r/makeupaddiction and r/wow, subreddits devoted, respectively to makeup 294 tips and the popular videogame 'World of Warcraft.' Further, this includes 295 only users who have taken the time to make a comment: many more 296 passively follow the forums. By the posting standards of Reddit, there- ${ }_{297}$ fore, $r$ /conspiracy is comparable to forums discussing topics of broad 298 mainstream interest rather than a tiny and isolated group.

\section{Language use: four interpretations}

Our study showed both commonalities and differences between subgroups 301 posting in $r$ /conspiracy. What, if anything, can one say about conspiracy 302 believers as a whole? We evaluate four classes of theory about conspiracy 303 endorsement in light of these findings. 
It is common to suppose that conspiracy endorsement is a sign of prima 306 facie irrationality. The idea of a 'monological belief system' is typically 307 introduced as the idea that belief in one conspiracy predicts belief in 308 different, apparently unrelated theories [13,26]. Many have noted the power 309 of conspiracy theories to give broad, unifying explanations at the expense of 310 plausibility [9]. This suggests a particular picture: the conspiracy theorist 311 is one who believes that everything is connected. 312

Yet we think that a move to attribute pathological irrationality would be 313 hasty. First, as we noted above, it is easy to overgeneralize from committed 314 posters. But second, and importantly, the strong correlation between length 315 and topic 0 suggests that posters are concerned above all with evidence and 316 argument. Contrary to the unflattering picture presented by many authors, 317 on which conspiracy endorsers are merely gullible or deluded, we note that 318 conspiracy theorists in fact spend an inordinate amount of time discussing 319 why they believe what they believe [2]. 320

Here we enter into delicate territory. There is a widespread assumption ${ }_{321}$ that the content of conspiracy theories is - perhaps by definition -irrational 322 to believe. Yet it cannot be irrational tout court to believe that the CIA ${ }_{323}$ meddles in international affairs, or that scientists are experimenting on 324 vulnerable populations without their consent, or that the US government 325 secretly monitors the activities of dissident groups. As True BeliEvers 326 are fond of pointing out, these things have all happened. The best one can 327 say is that a given event is not part of a conspiracy. 328

Further, as one of our sample quotes for the True BeliEvers suggests, 329 even voluminous posters often see themselves as discerning, rejecting as 330 many conspiracies as they accept. Tanya Luhrmann [27] noted a similar phe- 331 nomenon among practitioners of modern witchcraft, suggesting that among 332 marginalized communities internal debate is as important as agreement. $\quad{ }_{333}$

\section{First-order content}

Another possibility is that conspiracy believers are distinguished by par- 335 ticular sets of common beliefs. This is arguably the everyday picture - a 336 conspiracy theorist just is someone who believes in conspiracy theories, and 337 there is little more to say.

The diversity in our dataset makes it difficult to maintain this view, at 339 least if the relevant beliefs are meant to be ones shared by all conspiracy 340 theorists. The best candidate might be found among topic 34, concerning 341 US foreign policy, which correlates highly with comment length in all 342 subgroups. It is difficult to know which way the order of explanation goes, 343 however. The US is a powerful country; it may simply be a convenient 344 touchstone for a variety of otherwise different interests. 
Noteworthy is the ability of our method to distinguish between groups 346 like Anti-Authoritarians and Patriots. Both are concerned, in some 347 broad sense, with government violence and abuse of power. But they ${ }_{348}$ show different patterns of topic loading and different correlation patterns, 349 suggesting important distinctions between groups who may well discuss the 350 same events for superficially similar reasons.

Another (not exclusive) possibility is that conspiracy theorizing is driven 353 largely by what we will call second-order concerns: that is, concerns about 354 how one arrives at first-order conclusions. Wood et al [26] suggest that 355 learning about one conspiracy may encourage belief in others because 'Even 356 though the perpetrators may be different in each case, the fact that one ${ }^{357}$ massive, sinister conspiracy could be successfully executed in near-perfect 358 secrecy suggests that many such plots are possible.' The conspiracy theories 359 so endorsed need not bear any particular relationship to one another. Indeed, 360 it is possible that they might be mutually inconsistent. Wood et al. [26] 361 note a positive correlation between endorsing the statement that Osama 362 Bin Laden was dead before US soldiers arrived and endorsing the statement 363 that he is still alive. One might have thought those to be difficult beliefs to 364 hold simultaneously. However, this correlation is mediated entirely by a 365 more general belief that officials are engaged in a cover-up of some sort or 366 other.

The idea that some sources of information are unreliable cannot be an ${ }_{368}$ intrinsically irrational belief. As recent discussions of 'fake news' emphasize, 369 everyone believes that at least some media sources are systematically 370 distorted, and that epistemic responsibility thus involves keeping track 371 of which are reliable. Indeed, we find substantial evidence that posters 372 in $r$ /conspiracy are concerned with general problems about epistemic 373 responsibility. Recall that topic 0 - dealing with evidence and argument- 374 correlates strongly with log comment length for all subgroups. This suggests 375 that the more authors talk, the more they care about evidence, belief and 376 argument.

\section{Rhetorical features}

The above mostly concerns what endorsers believe. Yet as Hofstadter's [2] 379 classic work put it, 'It is the use of paranoid modes of expression by more 380 or less normal people that makes the phenomenon significant.' A fourth 381 possibility, therefore, is that conspiracy endorsement is a function of style 382 as much as substance. 383

The most obvious example of this are the InDIGNANT, who write short, 384 profanity-heavy posts. Indeed, the INDIGNANT raise an interesting pos- 385 
sibility: some posters might endorse a conspiracy theory without fully 386 believing it, as a way to express their background commitments. Someone 387 might endorse the statement 'The US was behind $9 / 11$,' not because they ${ }_{388}$ have positive beliefs about what happened, but as a way to vent their 389 anger about US domestic policy. Racist conspiracy theories may play a 390 similar role. Similarly, the REDDITORS seem to be as much concerned with 391 interpersonal drama on the site as they are with any particular conspiracy 392 theory. $\quad 393$

Finally, rhetorical topics may play a moderating role. Our methods are 394 relatively coarse-grained, and within groups such TRUTHERS, there can be 395 considerable disagreement [14]. Among our TRUTHERs, topics such as 4, 5, 396 14, and 17 correlate well with topic length and load higher than average. 397 These topics contain words that involve (among other things) moderating 398 inter-group disagreement, providing evidence that such groups are diverse 399 even internally.

\section{Limitations and future directions}

Several features and limitations of the present study are worth noting. $\quad{ }_{402}$

Topic analysis does not distinguish between people who endorse a con- ${ }_{403}$ spiracy (in any sense) and those who argue with conspiracy theorists. 404 Automated methods such as sentiment analysis for teasing out agreement 405 and disagreement would be a useful addition to the present method. $\quad 406$

More broadly, we formed subgroups solely by looking at language usage. 407 Other groupings are possible with the reddit dataset: one might look at ${ }_{408}$ network structure both within and outside of $r$ /conspiracy, for example, 409 by looking at relationships of mutual commenting. Examination of the ${ }_{410}$ network structure of other forums [3 5] has given important insight about ${ }_{411}$ the dynamics of problematic beliefs as they spread through social networks, ${ }_{412}$ and would be a useful compliment to the current investigation. Similarly, ${ }_{413}$ one might look at other places in which authors post, as an index of their ${ }^{414}$ overall interests outside r/conspiracy.

That said, it is also worth noting as a strength of the current study that 416 it is able to distinguish structure without relying on network considerations; ${ }^{417}$ the high mean neighbor diversity of each subgroup also suggests that ${ }_{418}$ different subgroups can find much to say to each other. $\quad 419$

Compared to other methods, NMF has a reputation for creating topics $\quad 420$ with more diverse quality [24] - the best topics are quite coherent and 421 consistent, while other topics are more diverse. However, we think that ${ }_{422}$ the present study offers a more nuanced view. Many 'rhetorical' topics ${ }_{423}$ are distinguished not by the pre-existing semantic similarity of the words ${ }^{424}$ to one another or their co-occurence per se, but rather by the fact that ${ }^{425}$ they distinguish similar ways of talking about other topics. Topic 44 (for ${ }_{426}$ 
example) contains a set of words pertaining to reading articles both in ${ }_{427}$ print and online. These need not co-occur, and may even partially exclude ${ }_{428}$ one another, while still representing an intuitively cohesive way of talking ${ }^{429}$ about evidence. That said, some of the topics have clear intruders (e.g. 430 topic 10, which mixes words about faked pictures and videos with those ${ }_{431}$ about actual incidents of fakery). More work is needed to distinguish low ${ }_{432}$ from high-quality topics, keeping in mind the compositional nature of NMF ${ }_{433}$ topics.

\section{Conclusion}

We have emphasized the degree to which conspiracy endorsers differ. We ${ }_{436}$ suspect that this diversity continues down to the level of motives as well. ${ }^{437}$ Theorists have suggested a wide variety of different motivations for belief ${ }_{438}$ in conspiracy theories, including the need for explanations, the desire ${ }_{439}$ for control in a complex world, political extremism, the desire for simple ${ }_{440}$ explanations, and so on. Some of these do not appear to be especially ${ }_{441}$ problematic motives per se. Indeed some form of low-grade conspiracy ${ }_{442}$ theorizing is widespread (consider the kvetching that academics do about ${ }_{443}$ the nefarious plans of administrators and granting bodies). $\quad{ }_{444}$

If we were to take r/conspiracy as a whole as a single author, that ${ }_{445}$ author would undoubtedly seem monological and paranoid. Yet very few ${ }_{446}$ authors within conspiracy fit a clearly monological pattern. We suggest that ${ }_{447}$ $r /$ conspiracy looks monological in part because there are many different ${ }_{448}$ authors with different sets of concerns, each interacting with one another. ${ }_{449}$ Consider a thread about (e.g.) secret CIA prison camps. One person ${ }_{450}$ might care about its relationship to $9 / 11$, another might use it to fuel their ${ }_{451}$ antisemitism, a third to make a point about gun control. Each gets what ${ }_{452}$ they need, and each contributes to the larger whole. ${ }_{453}$

Ultimately, we doubt that there needs to be any particular set of psy- ${ }^{454}$ chological motivations which characterize conspiracy theorists. Some are ${ }_{455}$ irrational. Some are irate. Some are epistemically unlucky. Some are 456 racist. Some are skeptical. We should not say that conspiracy theorists ${ }_{457}$ have overarching belief systems that encompass and unify a wide variety ${ }_{458}$ of different narratives. Instead, it may be the other way around: it is ${ }_{459}$ conspiracy narratives that are all-encompassing, pulling in a diverse group 460 of people who may have little in common with one another, each of whom ${ }_{461}$ can find what they need in a fragment of the larger tale. ${ }_{462}$ 


\section{Subgroup number}

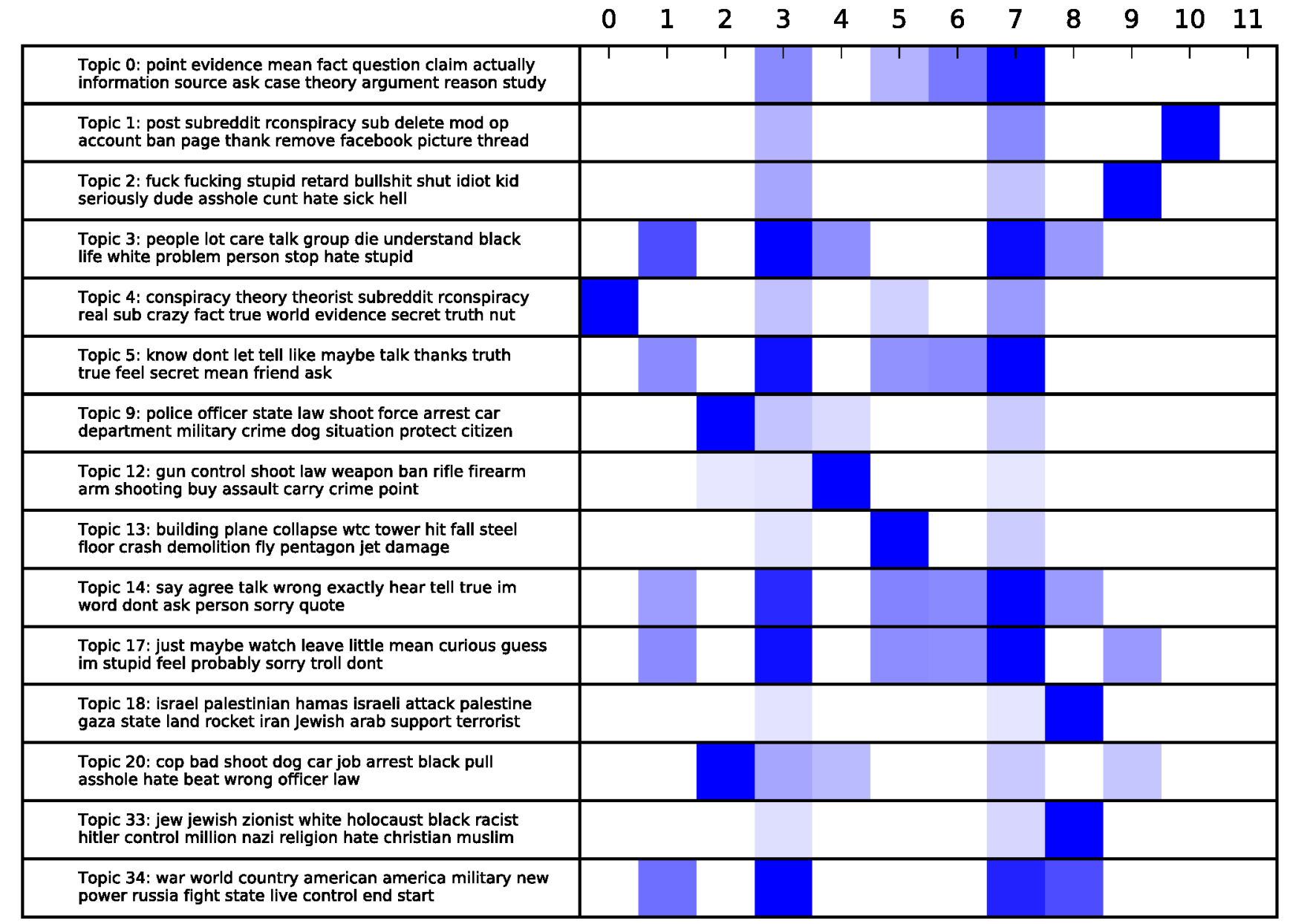

\begin{tabular}{|c|c|c|}
\hline \# & Name & Representative Quote \\
\hline 0 & SKEPTICS & $\begin{array}{l}\text { "This kind of thing comes up every month. Fuel burns differently when it is atomized than when it is in a puddle. Can } \\
\text { someone please explain why you guys keep falling for this conspiracy nonsense?" }\end{array}$ \\
\hline 1 & ANTI-IMPERALISTS & $\begin{array}{l}\text { "Boeing can already target anyone, anywhere. The US is a wholly owned and operated subsidiary of the military-industrial } \\
\text { complex. In a decade we'll have drones killing any American who disagrees with the slide into fascism." }\end{array}$ \\
\hline 2 & ANTI-AUTHORITARIANS & $\begin{array}{l}\text { "No doubt they are dirty cops and the store owner isn't paying up. 'Zero tolerance' is an excuse to shakedown business } \\
\text { owners. Police do whatever they want, they're a gang, no different from the Crips and the Bloods." }\end{array}$ \\
\hline 3 & True BeLIEVERS 1 & $\begin{array}{l}\text { "The difference is that the } 911 \text { truth movement has evidence on its side. The idea that Obama was born in Kenya is just a } \\
\text { Republican plot to distract people from health care reform. There is no evidence for it, and plenty of evidence against." }\end{array}$ \\
\hline 4 & PATriots & $\begin{array}{l}\text { "People who call for 'common sense' gun laws won't be happy until guns are banned. I'm sorry, but the bill of rights } \\
\text { protects the rights of the PEOPLE; Article 1, Section } 8 \text {, already authorizes the government to raise a standing army." }\end{array}$ \\
\hline 5 & TRUTHERS & $\begin{array}{l}\text { "Sorry guys, but you've clearly never watched the video. Anyone who sees the 'collapse' of WTC7 and still thinks it was } \\
\text { caused by fire rather than demolition is blind or a shill for the NIST." }\end{array}$ \\
\hline 6 & PSUEDOSCIENTISTS & $\begin{array}{l}\text { "I have read vaccine studies for } 20 \text { years. Why do you think they never use a control? It would make it obvious that } \\
\text { vaccines themselves spread highly profitable autoimmune diseases." }\end{array}$ \\
\hline 7 & True Believers 2 & $\begin{array}{l}\text { "If HAARP technology is indeed the superweapon so many say it is, then war between the superpowers may well have } \\
\text { begun in } 2010 \text {, and is being fought in secret. Fukishima was just another move in the game." }\end{array}$ \\
\hline 8 & ANTI-SEMITES & $\begin{array}{l}\text { "Or maybe you don't realize that the 'Arab Spring' was a Mossad/CIA plot from the start. Face it: only one nation is capable } \\
\text { of dragging the U.S. back into the Middle East while making it appear that Islam rather than Judaism is the problem."' }\end{array}$ \\
\hline 9 & INDIGNANT & "Fuck Israel. Or I maybe I should just keep my racist shit in my pocket - oh wait, it's already fucking full." \\
\hline 10 & REDDITORS & "The mods shadowbanned me 3 times in the last month alone. This subreddit is getting nuts." \\
\hline 11 & UNCATEGORIZED & "You don't have to 'produce' electricity, it's all around us. That's why it is called electricity." \\
\hline
\end{tabular}

Fig 1. Subgroup differences. Top: Subgroup loadings on selected topics. Topics represented by the top 15 words per topic. Loadings significantly above the mean are shown in blue $(p<0.01$ uncorrected). Loadings represented on a 0-1 scale, normalized per topic, with most saturated blue $=$ highest loading for that topic. Bottom: Subgroup, descriptive label, and aggregated quote (see text for details). 


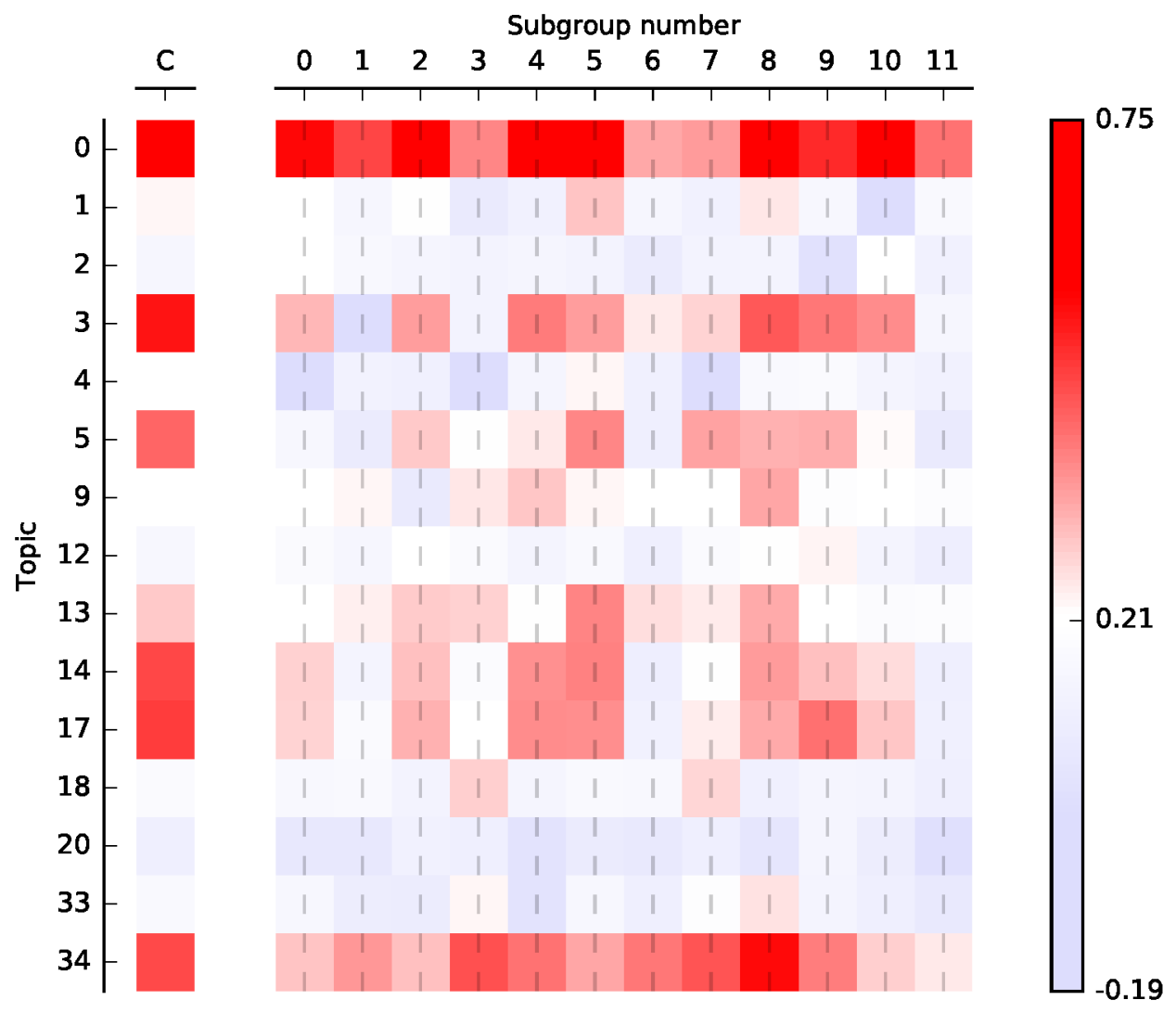

Fig 2. Correlations. Correlations between log comment length and loading for selected topics, corrected for overall correlation between mean loading and length. Correlations shown for conspiracy overall ('C') and for each of the 12 subgroups. Correlations significant to $p<.05$ uncorrected around the average random correlation of 0.21 . 


\section{References}

1. de Tocqueville A. Democracy in America. vol. 2. Nolla E, Schleifer JT, editors. Indianapolis: LIberty Fund; 1831.

2. Hofstadter R. The paranoid style in American politics. Harpers Magazine. 1964;229(1374):77-86.

3. Dunn AG, Leask J, Zhou X, Mandl KD, Coiera E. Associations between exposure to and expression of negative opinions about human papillomavirus vaccines on social media: An observational study. Journal of medical Internet research. 2015;17(6):e144.

4. Zhou X, Coiera E, Tsafnat G, Arachi D, Ong MS, Dunn AG, et al. Using social connection information to improve opinion mining: Identifying negative sentiment about HPV vaccines on Twitter. In: et al INS, editor. MEDINFO 2015: eHealth-enabled Health. Amsterdam: IOS Press; 2015. p. $761-765$.

5. Del Vicario M, Bessi A, Zollo F, Petroni F, Scala A, Caldarelli G, et al. The spreading of misinformation online. Proceedings of the National Academy of Sciences. 2016;113(3):554-559.

6. van der Linden S. The conspiracy-effect: Exposure to conspiracy theories (about global warming) decreases pro-social behavior and science acceptance. Personality and Individual Differences. 2015;87:171-173.

7. Goertzel T. Belief in conspiracy theories. Political Psychology. 1994;15(4):731-742.

8. Whitson JA, Galinsky AD. Lacking control increases illusory pattern perception. science. 2008;322(5898):115-117.

9. Keeley BL. Of conspiracy theories. The Journal of Philosophy. 1999;96(3):109-126.

10. Kahan DM. Climate-science communication and the measurement problem. Political Psychology. 2015;36(S1):1-43.

11. Raab MH, Ortlieb SA, Auer N, Guthmann K, Carbon CC. Thirty shades of truth: Conspiracy theories as stories of individuation, not of pathological delusion. Frontiers in psychology. 2013;4.

12. Bessi A, Zollo F, Del Vicario M, Scala A, Caldarelli G, Quattrociocchi W. Trend of Narratives in the Age of Misinformation. PloS one. 2015;10(8):e0134641. 
13. Swami V, Coles R, Stieger S, Pietschnig J, Furnham A, Rehim S, et al. Conspiracist ideation in Britain and Austria: Evidence of a monological belief system and associations between individual psychological differences and real-world and fictitious conspiracy theories. British Journal of Psychology. 2011;102(3):443-463.

14. Wood MJ, Douglas KM. Online communication as a window to conspiracist worldviews. Frontiers in psychology. 2015;6.

15. Leeman PJ. The born conspiracy. New Scientist. 2007;195:35-37.

16. Swami V, Voracek M, Stieger S, Tran US, Furnham A. Analytic thinking reduces belief in conspiracy theories. Cognition. 2014;133(3):572-585.

17. Van Prooijen JW, Krouwel AP, Pollet TV. Political extremism predicts belief in conspiracy theories. Social Psychological and Personality Science. 2015;6(5):570-578.

18. De Choudhury M, Kiciman E, Dredze M, Coppersmith G, Kumar M. Discovering shifts to suicidal ideation from mental health content in social media. In: Proceedings of the 2016 CHI Conference on Human Factors in Computing Systems. ACM; 2016. p. 2098-2110.

19. Bird S, Klein E, Loper E. Natural language processing with Python. O’Reilly Media, Inc.; 2009.

20. Miller GA. WordNet: A lexical database for English. Communications of the ACM. 1995;38(11):39-41.

21. Pedregosa F, Varoquaux G, Gramfort A, Michel V, Thirion B, Grisel O, et al. Scikit-learn: Machine Learning in Python. Journal of Machine Learning Research. 2011;12:2825-2830.

22. Lee DD, Seung HS. Learning the parts of objects by non-negative matrix factorization. Nature. 1999;401(6755):788-791.

23. Boutsidis C, Gallopoulos E. SVD based initialization: A head start for nonnegative matrix factorization. Pattern Recognition. 2008;41(4):13501362.

24. Stevens K, Kegelmeyer P, Andrzejewski D, Buttler D. Exploring topic coherence over many models and many topics. In: Proceedings of the 2012 Joint Conference on Empirical Methods in Natural Language Processing and Computational Natural Language Learning. Association for Computational Linguistics; 2012. p. 952-961.

25. Von Luxburg U, Williamson RC, Guyon I. Clustering: Science or art? In: ICML Unsupervised and Transfer Learning. vol. 27; 2012. p. 65-80. 
26. Wood MJ, Douglas KM, Sutton RM. Dead and alive beliefs in contradictory conspiracy theories. Social Psychological and Personality Science. 2012;3(6):767-773.

27. Luhrmann TM. Persuasions of the witch's craft: Ritual magic in contemporary England. Cambridge: Harvard University Press; 1991. 


\section{Supporting information}

\section{S1 Appendix}

\section{Ethics Statement}

Ethics approval was waived as we used only publicly available comments harvested via the Reddit API. All comments to the $r$ /conspiracy forum were publicly available at the time of data collection (first quarter of May 2015). The 'sample comments' in the main text were deliberately obfuscated to prevent their attribution to a particular user.

\section{Dataset}

We used a dataset made publicly available by the user 'Stuck_In_The_Matrix', who used the official reddit API to scrape a set of roughly 1.7 billion comments and associated metadata spanning from October 2007 to May 2015. (For more details see https://www.reddit.com/r/ datasets/comments/3bxlg7/i_have_every_publicly_available_reddit_comment/). The set has nearly every public reddit comment made in the time period. This includes nearly 2.25 million comments to $\mathrm{r}$ /conspiracy, made by nearly 130,000 distinct authors.

\section{Preprocessing}

Reddit allows posts by small automated programs known as 'bots', which can skew descriptive statistics. To remove account names associated with bots, we first looked at each poster in a target set of subreddits (including $\mathrm{r}$ /conspiracy) and calculated the number of other subreddits in which they posted (their forum diversity). A list was compiled of usernames whose forum diversity was more than 15 standard deviations above the mean. Manual inspection revealed that every member of this list was probably a bot, whereas more aggressive cuts also included posters who were clearly human. This was combined with a list of usernames corresponding to known bots posted on reddit itself. (From https://www.reddit. com/r/botwatch/comments/1xojwh/list_of_320_reddit_bots/) The 466 authors in the set were excluded from subsequent analyses. This procedure erred on the side of including bots rather than eliminating human posters. While the remaining set contains some bots, the combination of forum diversity and known bots eliminates the vast majority of automated posting, and the remaining bots should make for only a minor skew on the descriptive statistics. Of note, the remaining bots (such as there are) seem to be concentrated in the 'noisy' subgroup 11, about which we make no specific claims.

After extracting the comments in $r$ /conspiracy from the larger dataset, the comment text itself was preprocessed in several steps. Comments with authors or text bodies which had been subsequently deleted were removed. Whole lines which were preceded by markdown quotation indicators were removed, as were URLs and formatting escape codes were also removed. Words were then tokenized and converted to lowercase, and punctuation and non-alphabetic characters were removed.

Words were tagged with part-of-speech using the python Natural Language Toolkit package (nltk, [19]). Functional words such as pronouns and determiners were removed, leaving 
only nouns, adjectives, verbs, adverbs, and cardinal numbers. What remained was then lemmatized with nltk using the wordnet lemmatizer [20]. Any comment fewer than 3 words after preprocessing was eliminated from further analysis. Note that, unlike many studies, we did not eliminate short words, as there were semantically meaningful worlds of two and three characters that clearly belong in the final topics.

Because we are interested in drawing inferences about authors rather than individual comments, each author's comments were combined into a single document. The resulting corpus was transformed into a term frequency-inverse document frequency (tf-idf) representation. A tf-idf representation represents individual documents as a collection of normalized word frequencies, each weighted by the overall frequency of the word in the corpus. The weighting means that words which are extremely common are given relatively little weight, while words which distinguish a particular document are weighted more. At this step any remaining common english stopwords and words that appeared fewer than 20 times in the corpus were also eliminated, leaving a final vocabulary of 30,327 words. (This and subsequent steps used scikit-learn, [21]).

\section{Data Sharing}

Processed data for $r$ /conspiracy is available as a $165 \mathrm{MB}$ archive at: https://cloudstor.aarnet.edu.au/plus/index.php/s/GRjLJxoxN99RIJe

To ensure anonymity, the processed data contains neither author names nor identifiable comments. 


\section{S1 Fig.}

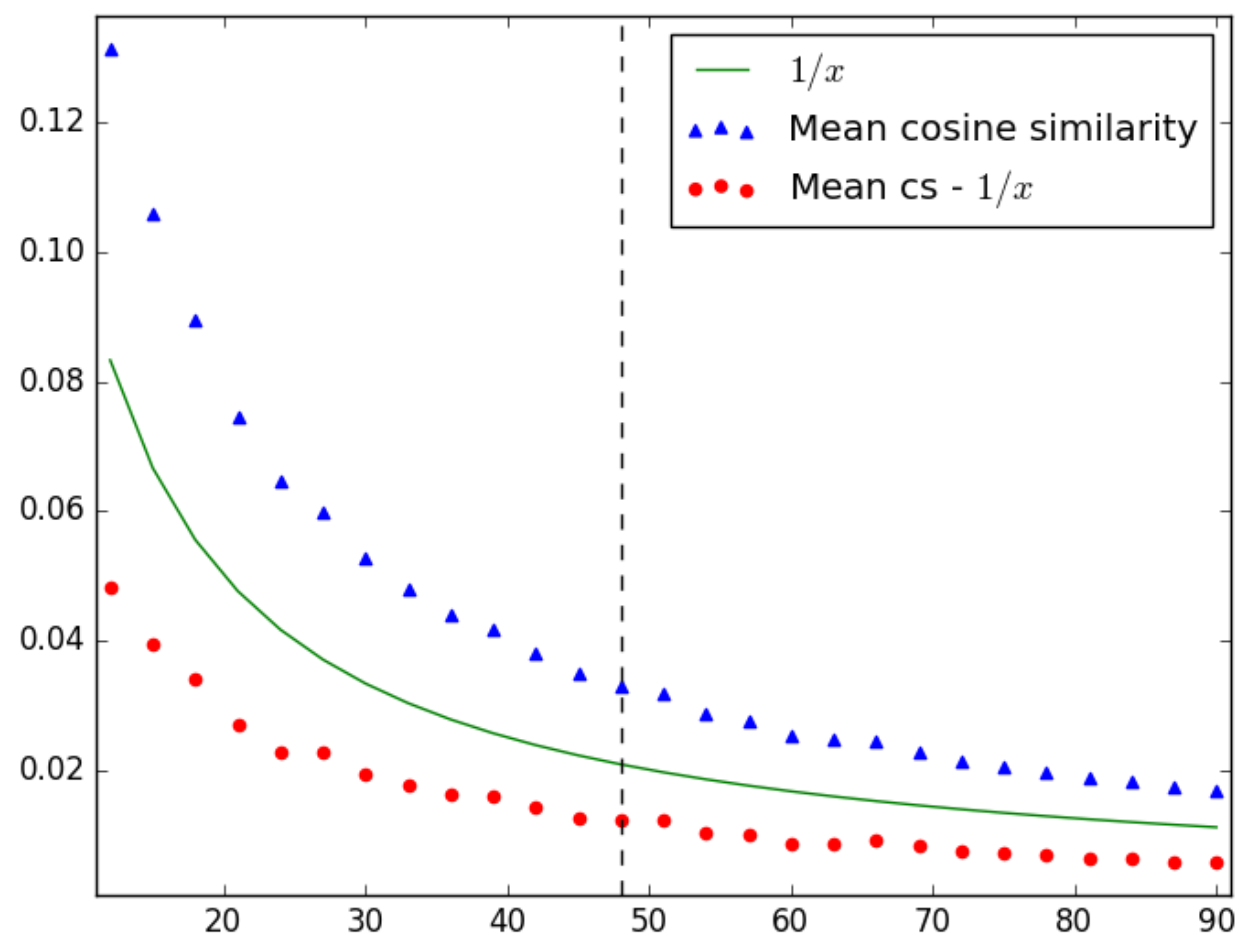

S1 Fig.: Mean pairwise cosine similarity for each vector in the topic-word mapping for a range of NMF topic numbers $12,15, \ldots 90.1 / x$ represents the theoretical lower bound of this value. Red circles indicate difference from the theoretical lower bound. Chosen topic number of 48 indicated by dashed line. 


\section{S1 Table}

0 point evidence mean fact question claim actually information source ask case theory argument reason study

1 post subreddit rconspiracy sub delete mod op account ban remove page facebook thank picture thread

2 fuck fucking stupid retard bullshit shut idiot kid seriously dude asshole cunt hate sick hell

3 people lot care talk group die understand black life white problem person stop hate stupid

4 conspiracy theory theorist subreddit rconspiracy real sub crazy fact true world evidence secret truth nut

$5 \quad$ know dont let tell like maybe talk thanks truth true feel secret mean friend ask

6 video watch youtube fake camera saw footage game film minute explain view release documentary second

7 good bad job idea point luck great thank thanks damn feel movie evil sir stuff

8 guy picture talk hey shoot bomb crazy backpack run black actually lol stuff idiot white

9 police officer state law shoot force arrest car department military crime dog situation protect citizen

10 look picture photo fake image eye pic backpack maybe edit lot face left white bomb

11 government control citizen american federal power law state medium public terrorist corporation secret protect corrupt

12 gun control shoot law weapon ban rifle firearm arm shooting buy assault carry crime point building plane collapse wtc tower hit fall steel floor crash demolition fly pentagon jet damage say agree talk wrong exactly hear tell true im word dont ask person sorry quote time long year day waste start hear watch spend hour tell ago live old new really wow care stuff hope lot interesting big kind feel bad like watch thanks understand just maybe watch leave little mean curious guess im stupid feel probably sorry troll dont israel palestinian hamas israeli attack palestine gaza state land rocket iran Jewish arab support terrorist read book interesting thank title write great actually thanks story stop stuff sound watch remember cop bad shoot dog car job arrest black pull asshole hate beat wrong officer law right wrong law freedom realize left amendment sound wing leave constitution speech citizen mean free believe tell god truth hard lie story actually alien true religion theory crazy fake idiot need help start stop change new feel page power upvotes dont let attention problem revolution link thanks source click page site google thread provide share thank website info original check make sense feel sick point fun difference statement sound bad decision wonder mistake person sad kill child kid year die shoot innocent death terrorist dog dead murder civilian school family think mean maybe agree exactly joke dont crazy idea probably word honestly big lot thought happen year day actually event story holocaust ago let probably wait week attack remember exactly want live hear exactly dont truth talk ask help change let free watch stop question comment thread delete sub account mod ban edit shill subreddit rconspiracy reply op page user come day mind hear truth hope year true let wait new start tell alien home sure pretty im lot actually probably sound joke big funny talk stuff lol dude hear jew jewish zionist white holocaust black racist hitler control million nazi religion hate christian muslim war world country american america military new power russia fight state live control end start use word water phone weapon google drug force internet site data term technology chemical company vote party obama paul election president candidate ron republican voting change count voter win democrat reddit mod sub ban user account page site admins subreddit new subreddits remove rconspiracy censor thing bad lot kind sort change different hear exact funny probably life stuff important learn yeah oh lol sound mean probably hear totally hell stuff haha dude definitely wait idiot money pay tax bank year company buy dollar debt gold business loan free market spend way feel life change best easy long start let help power agree live possible great man love god oh thank life black great thanks lol live hear white yes real news source story fox medium report watch hear cnn old tv site day page mainstream article year old ago write title source mention author website wikipedia quote original google publish work job day hard year live school company hour great tell kid life week home try nice stop help hard tell start shill let troll understand maybe sound hide figure shit holy fucking piece real stupid bullshit crazy lol oh dont dude dumb im sub

S1 Table: 48-topic NMF model for conspiracy. Showing top 15 words for each topic. 


\section{S2 Fig.}

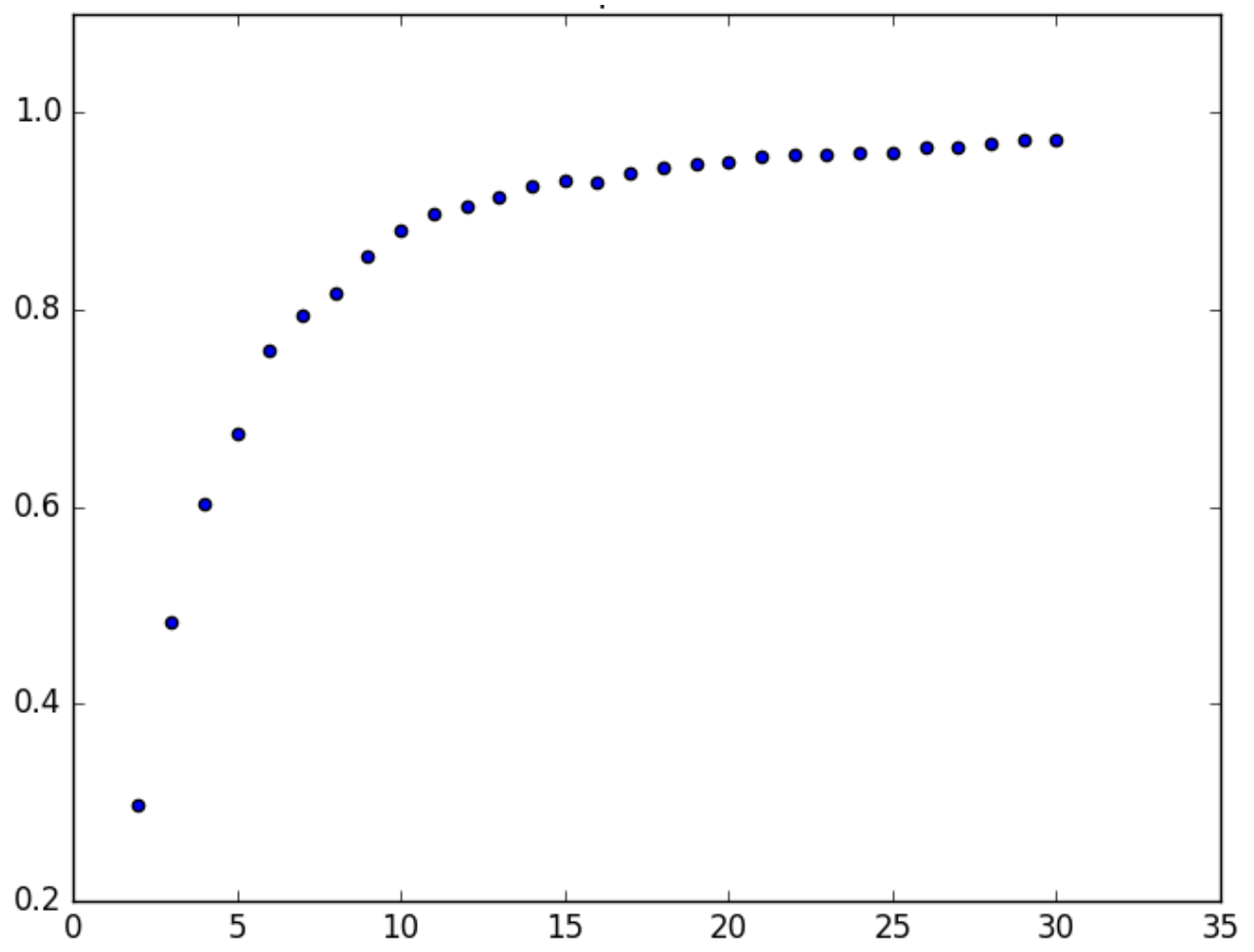

S2 Fig.: Percent variance explained for a range of kmeans clustering solutions. 


\section{S2 Table}

\begin{tabular}{|c|c|c|c|c|c|c|c|c|c|c|c|c|c|}
\hline Topic $\quad$ Group & $\mathrm{C}$ & 0 & 1 & 2 & 3 & 4 & 5 & 6 & 7 & 8 & 9 & 10 & 11 \\
\hline 0 & 0.73 & 0.56 & 0.47 & 0.62 & 0.38 & 0.61 & 0.75 & 0.34 & 0.35 & 0.64 & 0.51 & 0.6 & 0.41 \\
\hline 1 & 0.23 & 0.22 & 0.15 & 0.2 & 0.08 & 0.13 & 0.3 & 0.15 & 0.13 & 0.25 & 0.16 & -0.01 & 0.17 \\
\hline 2 & 0.16 & 0.22 & 0.17 & 0.15 & 0.14 & 0.15 & 0.14 & 0.09 & 0.14 & 0.14 & 0.03 & 0.22 & 0.13 \\
\hline 3 & 0.54 & 0.32 & -0.19 & 0.35 & 0.14 & 0.4 & 0.35 & 0.25 & 0.28 & 0.44 & 0.4 & 0.37 & 0.15 \\
\hline 4 & 0.22 & -0.0 & 0.13 & 0.12 & -0.03 & 0.15 & 0.23 & 0.12 & -0.08 & 0.17 & 0.18 & 0.14 & 0.12 \\
\hline 5 & 0.43 & 0.16 & 0.09 & 0.29 & 0.2 & 0.25 & 0.38 & 0.11 & 0.35 & 0.33 & 0.33 & 0.23 & 0.08 \\
\hline 9 & 0.21 & 0.21 & 0.23 & 0.08 & 0.25 & 0.3 & 0.23 & 0.21 & 0.21 & 0.34 & 0.2 & 0.21 & 0.19 \\
\hline 12 & 0.16 & 0.18 & 0.16 & 0.22 & 0.18 & 0.15 & 0.18 & 0.11 & 0.18 & 0.2 & 0.23 & 0.15 & 0.11 \\
\hline 13 & 0.29 & 0.22 & 0.24 & 0.29 & 0.28 & 0.2 & 0.39 & 0.26 & 0.25 & 0.34 & 0.21 & 0.19 & 0.19 \\
\hline 14 & 0.47 & 0.28 & 0.15 & 0.3 & 0.18 & 0.37 & 0.39 & 0.11 & 0.2 & 0.35 & 0.31 & 0.27 & 0.11 \\
\hline 17 & 0.48 & 0.28 & 0.18 & 0.32 & 0.22 & 0.37 & 0.37 & 0.13 & 0.24 & 0.33 & 0.41 & 0.3 & 0.12 \\
\hline 18 & 0.18 & 0.17 & 0.17 & 0.14 & 0.29 & 0.15 & 0.17 & 0.17 & 0.28 & 0.12 & 0.15 & 0.14 & 0.11 \\
\hline 20 & 0.11 & 0.07 & 0.07 & 0.13 & 0.11 & 0.04 & 0.09 & 0.08 & 0.11 & 0.06 & 0.15 & 0.1 & 0.03 \\
\hline 33 & 0.18 & 0.16 & 0.1 & 0.1 & 0.23 & 0.04 & 0.16 & 0.11 & 0.2 & 0.26 & 0.15 & 0.11 & 0.08 \\
\hline 34 & 0.47 & 0.3 & 0.36 & 0.3 & 0.46 & 0.41 & 0.34 & 0.41 & 0.45 & 0.56 & 0.4 & 0.28 & 0.25 \\
\hline
\end{tabular}

Raw numeric correlations between selected topics and log length; corresponds to Fig. 2 in main text. 